\title{
OBSERVATION OF SOLAR FLARE TYPE PROCESSES IN THE LABORATORY
}

\author{
W. H. BOSTICK, V. NARDI, L. GRUNBERGER and W. PRIOR \\ Stevens Institute of Technology, Hoboken, N.J., U.S.A.
}

\begin{abstract}
A filamentary magnetic structure is produced on the plasma current sheath of a coaxial accelerator operated with deuterium. Space and time analysis of X-ray, neutron and visible-light emission indicates that the magnetic energy of the filaments is transferred to the plasma during a process of decay of the filaments. X-ray photographs show very localized regions (diameter $<0.5 \mathrm{~mm}$ ) of strong emission. Some of these regions are also located where the plasma is not subject to a maximum of compression. Similar bright spots $(\mathrm{H} \beta)$ are observed by $5 \mathrm{~ns}$ image converter photographs. The detailed analytical description of the self-consistent fields is deduced. The localized regions of strong emission may well correspond with the explosive onset of an instability at a point on a filament (single filament decay) or at a point where two filaments with opposite fields coalesce with magnetic field annihilation. The similarities with solar flares are considered.
\end{abstract}

\section{Experimental Technique and Observations}

The idea proposed by several authors (Gold and Hoyle, 1960; Alfvén and Carlquist, 1967), that a substantial amount of energy can be transferred from magnetic field to plasma at a very high rate (as in solar flares) is tested with laboratory data.

In order to produce the plasma with a suitable magnetic structure, we have used a coaxial accelerator (CA) with electrode radii $5 \mathrm{~cm}$ and $1.7 \mathrm{~cm}$ - center electrode positive - connected at one end (breach) to a $45 \mu F$ capacitor bank initially at a potential $V=13 \mathrm{kV}$. This device has been extensively described in the literature (Marshall, 1960); some data are recalled here. The cylindric coaxial electrodes (see Figure 1) are in a vacuum system containing deuterium at a well defined initial pressure $p$. With the onset of the discharge at the breach, a current sheath $(C S) \approx 1 \mathrm{~cm}$ thick carrying most of the current $(\approx 90 \%)$ through the plasma, moves between the electrodes with a velocity $u_{0}=u_{0}(p, V, \ldots)$ from the breach to the other end (muzzle) of $C A$. $C S$ is driven in the axial direction by the force density $\mathbf{j} \times \mathbf{B} ; \mathbf{j}$ is the current density through the plasma on $C S$ and $\mathbf{B}$ is given essentially by the magnetic field $B_{\theta} \approx|\mathbf{B}|$ in the plasma region behind $C S$ due to the current $I$ in the electrodes.

By magnetic probe measurements and image-converter $(I C)$ photographs taken at specific times, it is verified that the thin layer $(\approx 3 \mathrm{~mm}$ thick $)$ which carries about half of all the current on the sheath, contains the plasma front $\approx 1 \mathrm{~mm}$ thick which strongly radiates in the visible spectrum. By $C S$, we indicate from now on specifically this luminous layer.

The fine structure of $C S$ is better observed by choosing the initial value of the $C A$ parameters $(p, V$, etc.) in a specific interval. Our $C A$ is usually operated with deuterium $p=8$ torr, peak value of the electric current across $C A I_{\max } \approx 0.5 \mathrm{Ma}$. Then $C S\left(u_{0} \approx 4.0 \times 10^{6} \mathrm{~cm} / \mathrm{s}\right)$ is observed to be composed largely of luminous plasma filaments (diam $0.3-0.5 \mathrm{~mm}$ ) oriented in the radial direction, i.e. orthogonal to $\mathbf{B}_{0}$ 
direction (see Figure 2; optimum conditions for filament observation are found at $V=11-13 \mathrm{kV})$.

By decreasing $p$ (e.g. down to 0.6 Torr) at a constant $V=13 \mathrm{kV}, I C$ photographs of $C S$ do not readily show a filamentary structure by visible light whereas simultaneous observations of the electron density gradient $\left|\nabla \varrho_{-}\right|$by Schlieren shadowgrams show a

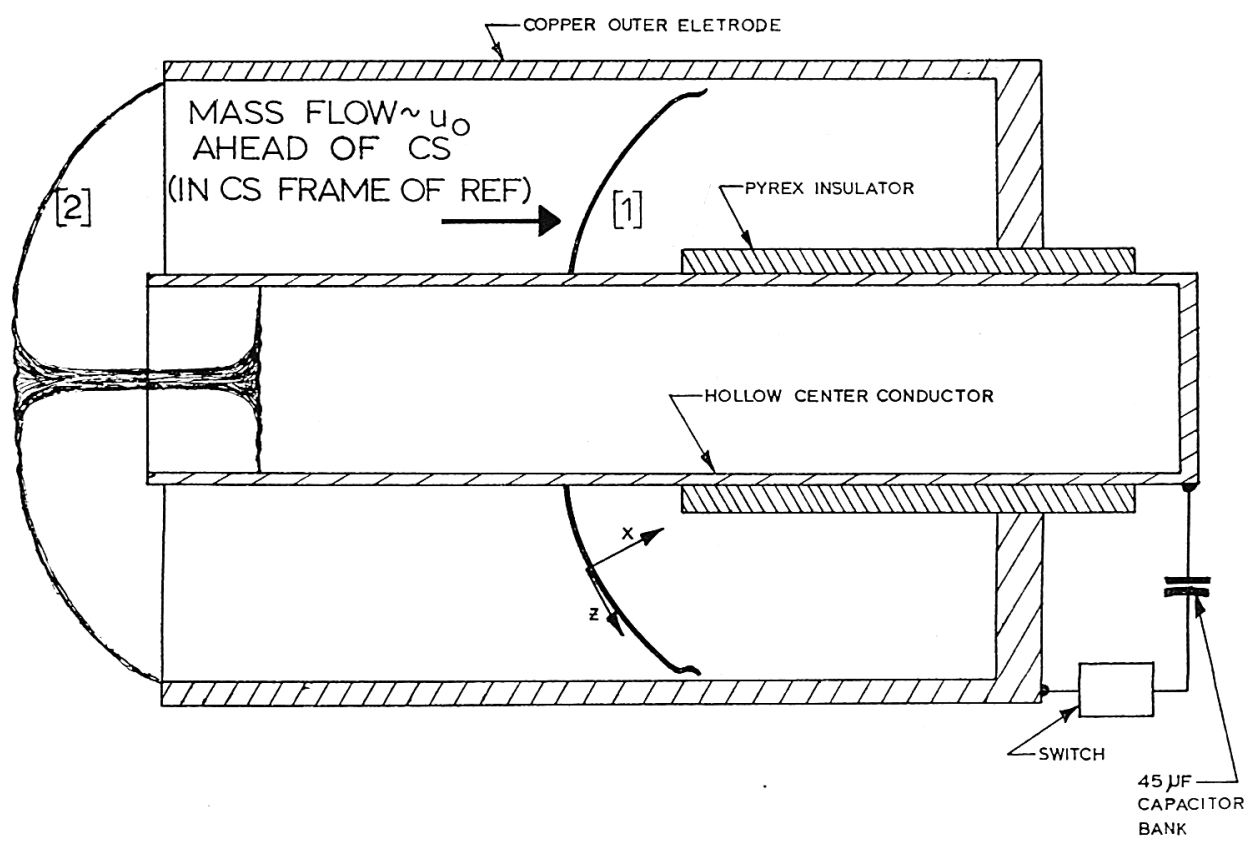

Fig. 1. Cross-sectional view of the $C A$. Current sheath between the electrodes [1] and at the later stage of axial collapse [2].
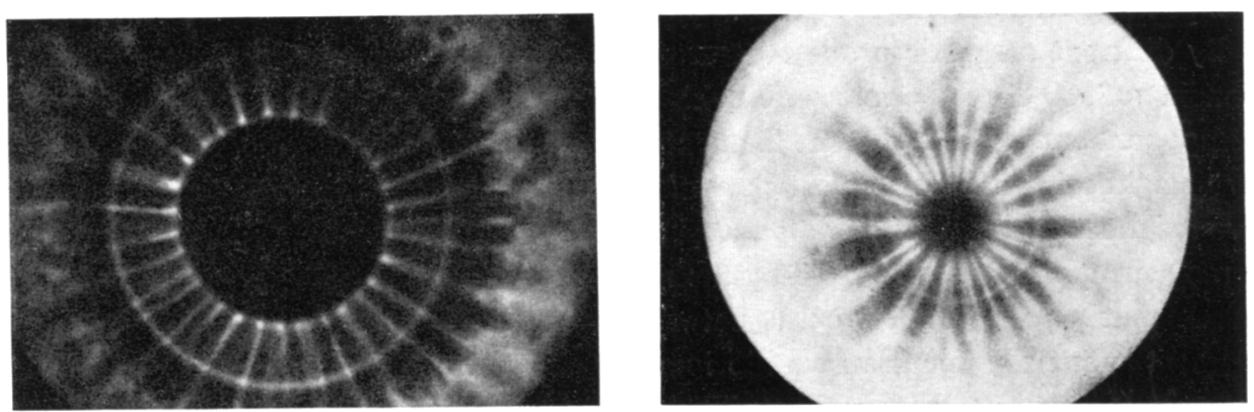

Fig. 2. Left: Front view of $C S$ with filaments rolling off the end of the center electrode. Imageconverter photos by visible light; $5 \mathrm{~ns}$ exposure (at $t=-120 \pm 20 \mathrm{~ns}$ before time of maximum axial compression). The circular edge of the center electrode (diam $3.4 \mathrm{~cm}$ ) is visible in the background $\left(D_{2}\right.$ initial pressure 8 Torr; peak value of electric current $0.5 \mathrm{Ma}$; applied potential $\sim 13 \mathrm{kV}$ ). Right: Same conditions but 100 ns exposure. A relevant fact is the absence of azimuthal motion of the filaments on $C S$. No blurring of the filament profile is observed with the long exposure if compared with $5 \mathrm{~ns}$ exposure. This fact demonstrates the permanent, stable character of the filamentary structure of $C S$. 
filamentary pattern. An apparently uniform $C S$ by visible light is also observed by increasing $V$ to sufficiently high values. A statistical analysis on many discharges indicates that the average number of radial filaments for a given $p$ increases by increasing $V$ (see Table I). $C S$ and filament arrangement on $I C$ photos remain substantially unchanged by using an interference filter with a $100 \AA$ band width and transmission peak near the $\mathrm{H} \beta$ line. Spectrograph data confirm that a major percentage of the visible light is emitted near $\mathrm{H} \beta$. The surface $\Sigma$, defined as the foremost luminous face of $C S$, is a direct source of information on flow and current distribution on $C S$. As a consequence of the observed nonplanarity and specific curvatures of $\Sigma$, one can easily prove by using MFD shock equations that vorticity $\omega=\frac{1}{2} \boldsymbol{\nabla} \times \mathbf{u}$ is different from zero in the filament region immediately behind $\Sigma$ and is oriented in the same direction as the filament axis (Bostick et al., 1969). It is observed that the plasma density, plasma flow density and magnetic fields are concentrated in these filaments (vortex filaments) (Bostick et al., 1966, 1970). The electron density by $\mathrm{H} \beta$ Stark broadening in the filaments appears greater by a factor $\approx 3$ than in the near luminous region.

TABLE I

\begin{tabular}{lll}
\hline $\begin{array}{l}\text { Operating initial } \\
\text { conditions }\end{array}$ & $\begin{array}{l}\text { Average number of } \\
\text { radial filaments } \\
\text { before } C S \text { collapse }\end{array}$ & $\begin{array}{l}\text { Average number of } \\
\text { radial filaments } \\
\text { after } C S \text { collapse }\end{array}$ \\
\hline $9 \mathrm{kV}, 8 \mathrm{~mm} D_{2}$ & 28 & 16 \\
$11 \mathrm{kV}, 8 \mathrm{~mm} D_{2}$ & 38 & 23 \\
$13 \mathrm{kV}, 8 \mathrm{~mm} D_{2}$ & 44 & 34 \\
\hline
\end{tabular}

Probe data show that $B_{\theta}$ vanishes ahead of $C S$ which coincides with the region of quick transition to high $B_{\theta}\left(B_{\theta}\right.$ increases to $\approx 1.4 \times 10^{4} \mathrm{G}$ by crossing $C S$, up to $\approx 2 \times 10^{4} \mathrm{G}$ behind the whole current layer). $C S$ has a magnetic structure much more complicated than the simple $B_{\theta}$ field.

By different magnetic probe measurements, it is possible to detect a strong component $\approx 2500 \mathrm{G}$ of the magnetic fields along the filament axis. This magnetic field component vanishes outside the filaments and usually has an opposite orientation for two neighboring filaments (Bostick et al., 1966). The preference sometimes shown by two neighboring filaments for a parallel arrangement (instead of the radial, spokelike arrangement which is suggested by the axial symmetry of the whole system) directly shows that a pairing effect exists between two filaments. A pair of parallel vortex filaments normally keeps an equilibrium distance from one another for a relatively long time (Figure 2). When this equilibrium distance is upset (for example by a shock wave which occurs in the medium at a specific stage of the discharge) the vortex pair is observed to coalesce (presumably with appreciable magnetic field annihilation), the region of combination traveling along the vortex filaments with a speed $\approx 2-5 \times 10^{7}$ $\mathrm{cm} / \mathrm{s}$. When $C S$ reaches the muzzle, it rolls off the electrodes, comes around the edge of the center electrode, proceeds across the front face with a radial velocity 
$\approx 7.5 \times 10^{6} \mathrm{~cm} / \mathrm{s}$ (radial and axial velocities of $C S$ are found statistically by plotting the observed positions versus time for a number of discharges) and collapses on the $C A$ axial region in a cylindric column (diam $\approx 4-5 \mathrm{~mm}$ ) collinear with the electrode axis. The number of filaments decreases during this stage approximately by a factor $\frac{2}{3}$ (see Table I). This occurs mainly by two-by-two-filaments coalescence processes which ordinarily begin at the radially innermost point of the combining pair.

In correspondence with a coalescence process, a typical fork configuration of the filaments can be observed on the moving off-axis part of $C S$ where resolution of the structure remains possible. At the stage of maximum axial compression, the remaining filaments partially blend in the cylindrical pattern on the axial region (dense plasma focus) but in the off-axis part of $C S$ filaments can still be observed quite clearly by visible light (see Figure 3).

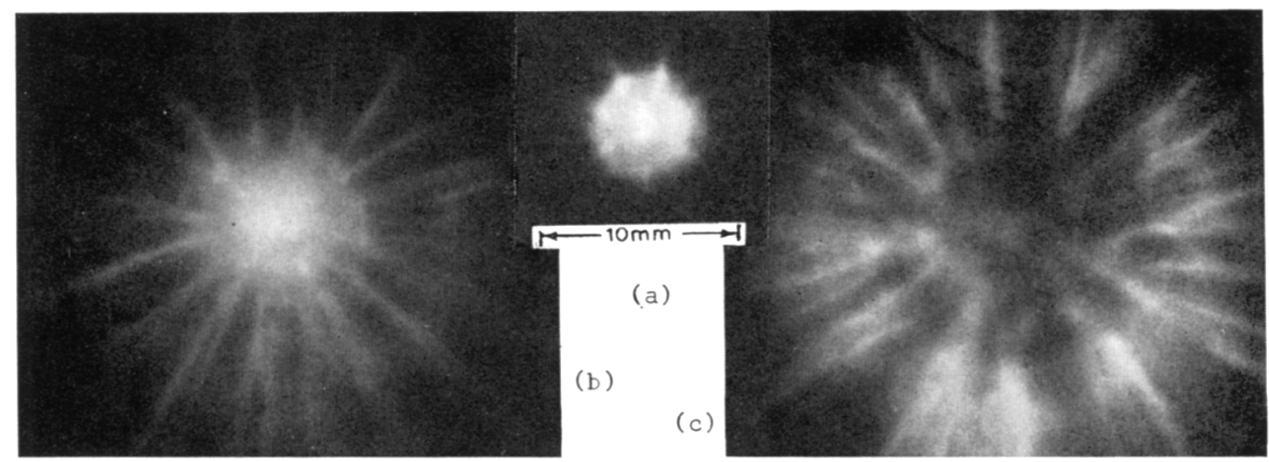

Fig. 3. $5 \mathrm{~ns}$ photos at different times from the time of maximum axial compression. (a) $t \sim 30 \mathrm{~ns}$; (b) $t \sim 100 \mathrm{~ns}$; (c) $t \sim 180 \mathrm{~ns}$. (a), (b) show clearly bright spots where filaments coalesce. Neutron production starts in (a); reaches its peak value in (b) and is still high $\left(\sim \frac{1}{3}\right.$ of the peak value) in (c) where no plasma concentration is shown by visible light in the axial region.

The stage of maximum compression, as shown by visible-light photos, occurs on the axial region starting from the end of the center electrode at a time coincident with a typical well-defined negative peak in the $\partial I / \partial t$ curve given by an oscilloscope trace. This peak time is conventionally taken as $t=0$. At $t=0$, a bright spot $(B S)$ appears on the front face of the center electrode and apparently moves on the axial column with a velocity of $\approx 2-5 \times 10^{7} \mathrm{~cm} / \mathrm{s}$. The off-axis part of $C S$ branches off immediately ahead of $B S$. A dark region in the column follows behind $B S$ which appears the region where the filaments coming together, start to coalesce and/or decay by high rate processes. The production of both X-rays $\left(\approx 1-10^{3} \mathrm{keV} ; \approx 95 \% \geqq 15 \mathrm{keV}\right)$ and neutrons starts almost simultaneously at $t \approx 20-40 \mathrm{~ns}$. The neutrons produced by $\mathrm{D}+\mathrm{D} \rightarrow \mathrm{He}^{3}+n+3.2 \mathrm{MeV}$ are detected in two ways, viz. by a silver activation counter (standard type developed at Los Alamos Sci Lab.) which gives a measure of the total number of neutrons per discharge and by a fast plastic scintillator (NE-102) which is connected via photomultiplier (RCA 5819) to an oscilloscope (rise time of whole detection system $\approx 20 \mathrm{~ns}$ ). A similar scintillator (but with a thinner plastic disk) 
is used for X-rays. The small thickness in this case insures against response to neutrons. Other methods based on neutron time-of-flight and on X-ray absorbing foils (aluminum) are used to separate neutron and photon signals also for calibration procedures. X-ray-time-integrated images of the off-electrode plasma region are also recorded by pin-hole camera photographs. About $90 \%$ of the X-ray signal appears to originate within $7.5 \mathrm{~mm}$ of the anode and are mainly due to the anode bombardement and anode vapor. The amplitude of this soft X-ray ( $\$ 10 \mathrm{keV})$ signal can be decreased to $\approx \frac{1}{10}$ by replacing the solid with a hollow center electrode (a pipe with a $3 \mathrm{~mm}$ thick wall). Unless specifically indicated, we will not refer to these electrode X-rays which can be easily discriminated from the X-ray produced in the deuterium plasma. Most of the X-rays which originate out in the plasma, away from the anode, have energies $\gtrsim 10 \mathrm{keV}$ far in excess of the electrode potential. The first X-ray burst is mainly due to this hard component. Since our $C A$ is operated near neutron threshold for a better resolution of the $C S$ structure, the total number of neutron $N$ produced in each discharge can fluctuate up to a factor $10^{4}$. The mean value is $10^{8}$ with an observed maximum of $\approx 4 \times 10^{8}$.

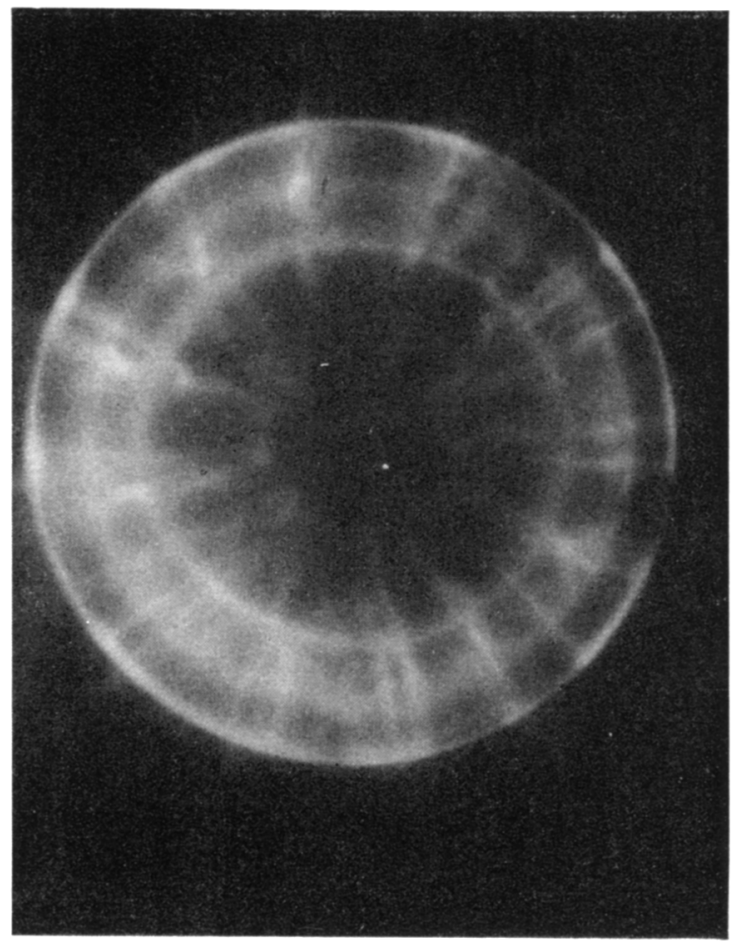

Fig. 4. Same conditions as Figure 2; $5 \mu$ s exposure at $t=250 \pm 20 \mu$ s. The glowing of the radial filaments in the off-axis region preceeds the decay induced by the radial shock. This correlated (shock-induced) decay of many filaments resembles features of the sympathetic flare phenomenon. The formation of a new kind of filament (rings) parallel to $B_{\theta}$ (related also to the slowing-down of $C S$ ) is evident. 
No appreciable variations in the neutron production are introduced by using the hollow anode (in this case $C S$ after collapsing in the axial region propagates inside the anode, with a filamentary structure quite similar to that occurring outside the anode; see Figure 1). In coincidence with the en mass-combination and decay of the vortex filaments in the axial column, the first $\Delta N / \Delta t$ pulse rises and reaches the peak value in $\approx 40-60 \mathrm{~ns}$, i.e. at the time $t=60-100 \mathrm{~ns}$ in which $B S$ has reached the end of the column. A clear indication exists that the dark region behind $B S$ where complete decay of the filament occurs, coincides with the region of X-ray and neutron production since this starts only when the dark region begins to grow between $B S$ and the center electrode.

Neutrons and X-rays are frequently emitted also in a later pulse at $t \approx 200 \mathrm{~ns}$ apparently in coincidence with the observed vortex filament coalescence (by pairs) and decay (by single filament processes) in the off-axis region of $C S$ when a disruptive shock wave, partially due to plasma ejected from the axial region, invests $C S$ and starts to propagate in the radial direction across $C S$ (see Figure 4). Overall, there is thus evidence indicating that the X-ray and neutron production is due to the decay of the filamentary magnetic structure of $C S$, in particular to the magnetic field annihilation associated with the combining of vortex filaments. This last decay process is essentially the solar flare process as proposed by Gold and Hoyle (1960).

\section{Particle Acceleration Mechanism}

Production of high energy electrons and ions and so of X-rays and neutrons at the focus is frequently explained in the literature (Mather, 1965) as the result of the plasma radial compression in the axial column due to the inward motion of $C S$ and to pinch effect ( $C A$ is operated with $I$ reaching $I_{\max }$ at the time of $C S$ collapse). Specifically two models which ignore the filamentary structure have been proposed to account for the neutron yield (Patou et al., 1969). It is now generally acknowledged in the literature that both those models cannot consistently explain the measured anisotropies of the neutron emission and the center of mass velocity $(1.2-2) \times 10^{8} \mathrm{~cm} / \mathrm{s}$ of the reacting deuterous as given by the neutron energy spectrum. (Patou et al., 1969). The resolution of the $C S$ structure in our experiments yield the information necessary for a more refined analysis of the focus stage. To summarize the essential role of the magnetic structure decay in the particle acceleration and ultimately in the plasma heating, we single out the following experimental observations:

(a) The different neutron pulses (frequently from one to three pulses are detected on an interval of time between 0.06 to $0.6 \mu$ s from $t=0$ ) cannot be explained simply in terms of successive pinchings of the plasma column. Second and third $\partial I / \partial t$ minima are occasionally observed. These minima are often - not always - followed by X-ray and neutron bursts but temporal correlation between all three signals cannot be established at the late $\partial I / \partial t$ peak times as it is for the first (compression) peak at $t=0$. The time separation between different neutron pulses is in a better agreement with the different times of decay of the filamentary structure observed in different regions 
on the plasma column and on the off-axis region of $C S$. These decay times can be obtained by observing the intense glowing of decaying filament segments (immediately before their disappearance) and of the forks in coalescence processes. The experimental evidence indicates that the giowing of a filament region is a precursor phenomenon $\approx 20$ to 100 ns ahead of the complete decay of the filamentary structure in that region.

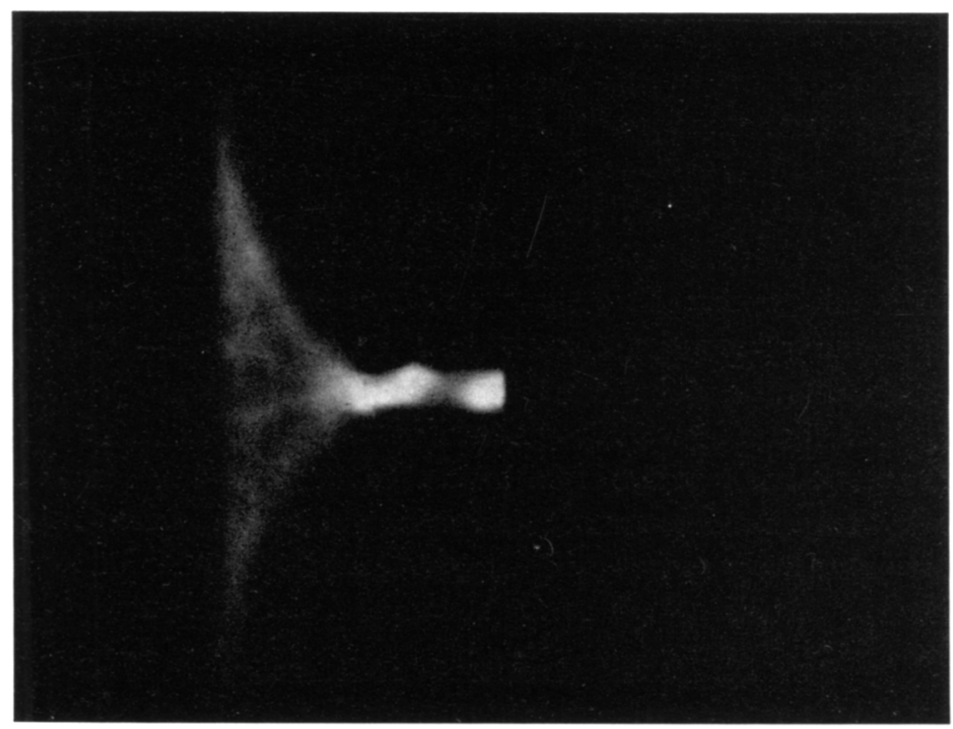

Fig. 5. Same conditions as Figure 2. Side view at $t=40 \pm 20 \mu \mathrm{s}$.

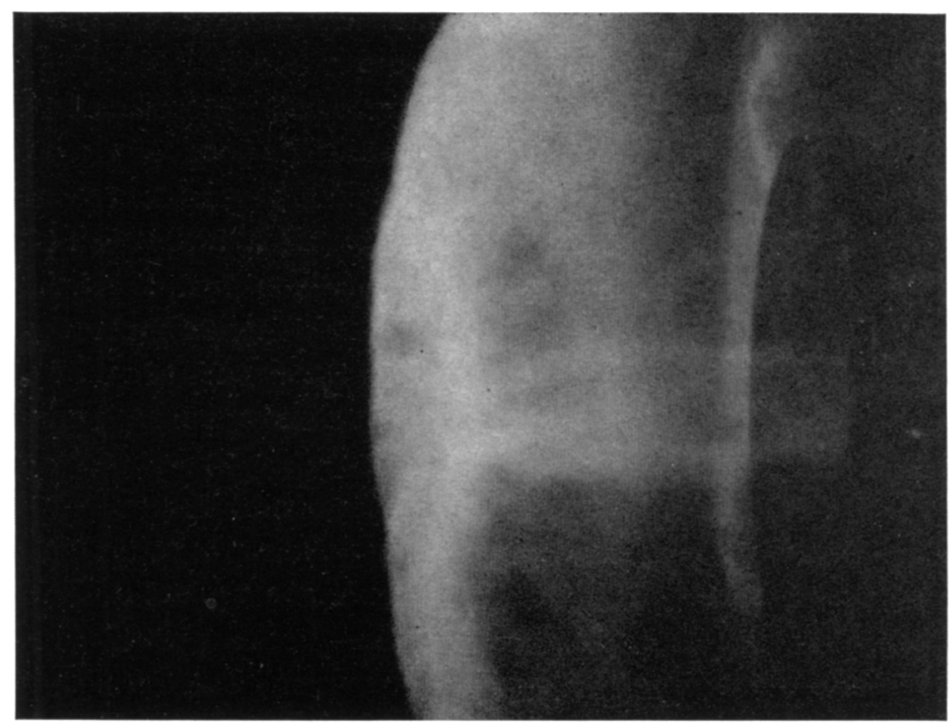

Fig. 6. Same conditions as Figure 2 but for the introduction of an axial magnetic field $\left(\approx 10^{3} \mathrm{G}\right)$ in the anode-front region. Side view at $t=100 \pm \mathrm{ns}$. The external axial field reduces the neutron yield by a factor $\approx 10^{-2}$. 

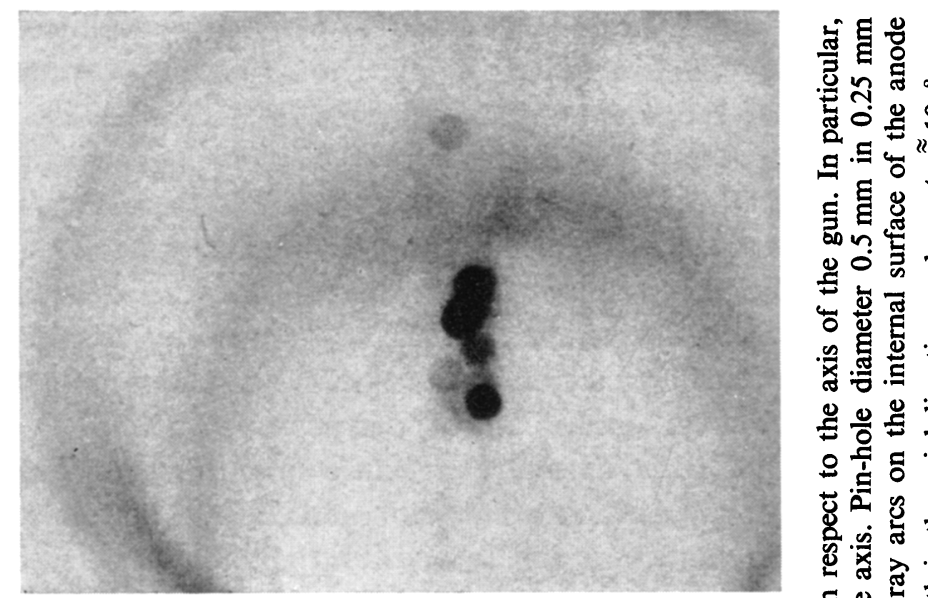

过

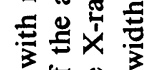

in

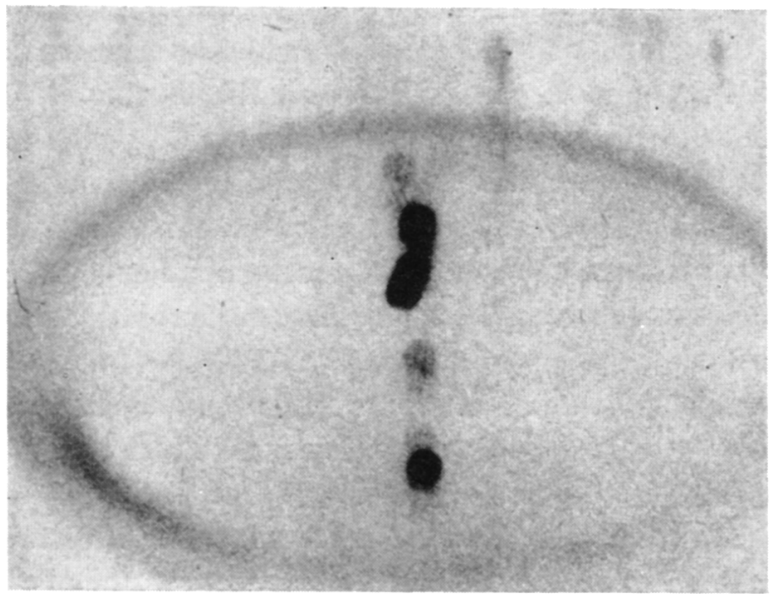

뭉

ส ธี 응

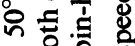

응 क

记 छ

它

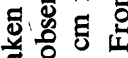

\& 8 :

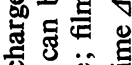

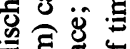

हㅕ

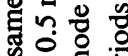

\& $V$ 呴

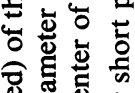

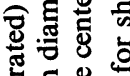

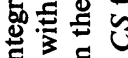

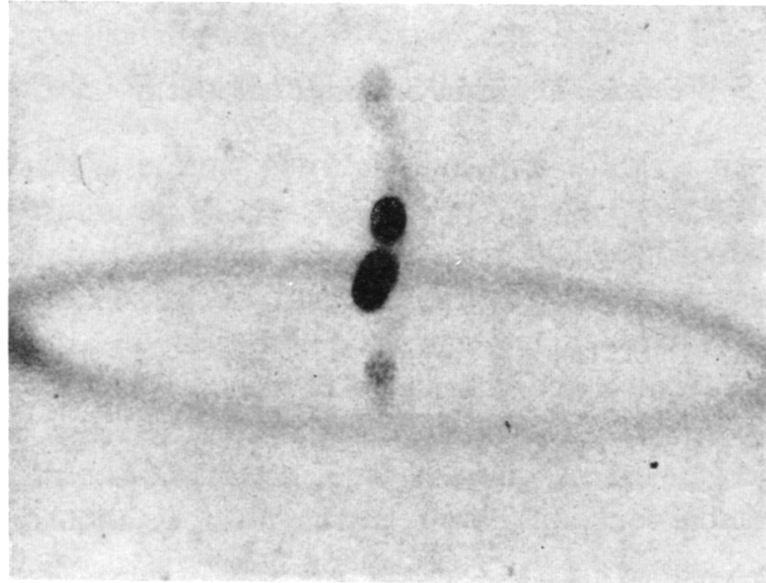

응 용

영 현

胥急

닌.

동ㅎㅀ

원.

로용

둥

행혈

本我氞

형휴

형용

명 응

근

일 
(b) Space-time resolution of the X-ray source is obtained by a collimator $(1.5 \mathrm{~mm}$ pass-through gap) orthogonal to the axial column. The hard X-ray generating region is apparently moving along the column with a velocity $\sim 3 \times 10^{7} \mathrm{~cm} / \mathrm{s}$. This is shown by the relative delay of the X-ray peak signals for two different locations (e.g. 13.5 and $26 \mathrm{~mm}$ ) of the collimator gap along the axial column. This velocity agrees with the velocity of $B S$ and with the apparent axial velocity of the neutron-generating region as determined by collimated-neutron measurements from other laboratories (Bernstein et al., 1969). Since a clear space-time correlation is so established among X-ray, neutrons and the filament decay precursor, it seems reasonable to conclude that the filament decay is the direct cause of $\mathrm{X}$-ray and neutron production.

(c) The importance of the filamentary structure in particle acceleration is also indicated by X-ray ( $\gtrsim 15 \mathrm{keV}$ ) pinhole photographs of $C S$ (front view, time integrated) which show a filamentary structure similar to visible light and Schlieren images (Lee et al., 1968). The fact that the filamentary structure exists in the axial column, where optical resolution is usually more difficult than for the previous stages of $C S$, is indicated by inhomogeneity and irregular shape of $B S$ on the column. Sometimes $B S$ is confined to a part of the column diameter (see Figure 5). The same irregular pattern is shown by side-view-X-ray pinhole photos. More specifically the existence of the filaments in the column can be proved by slowing down the $C S$ collapse in the axial region. This is accomplished by introducing an external magnetic field up to $\approx 10^{3} \mathrm{G}$ confined to the axial region in the direction of the electrode axis. The reaction of this field to the compression due to the collapsing $C S$, results ultimately in a greater diameter of the column at maximum compression so that the resolution of the column structure in terms of filaments remains clear also by $I C$ photos (see Figure 6). Our conclusion is that the magnetic structure decay propagates along the filaments with a velocity $2-5 \times 10^{7} \mathrm{~cm} / \mathrm{s}$. The corresponding inductive fields eject particles both in the radial and axial directions with a high energy which accounts for the $1.2-2 \times 10^{8} \mathrm{~cm} / \mathrm{s}$ center-of-mass velocity of the reacting deuterons. The magnetic structure decay can be of an explosive type in some localized region of a filament in the axial column as well as in the off-axis part of $C S$. This is proved by the localized intense X-ray sources located in different plasma regions as is shown by pinhole photos in Figure 7.

\section{Self-Consistent Fields. Structure and Decay}

The density in phase-space $f_{ \pm}$for ions and electrons satisfies $\mathrm{d} f_{ \pm} / \mathrm{d} t=S_{ \pm}$where $\mathrm{d} / \mathrm{d} t=\partial / \partial t+\mathbf{v} \cdot \nabla \pm e / m(\mathbf{E}+\mathbf{v} \times \mathbf{B}) \cdot \nabla_{\mathbf{v}}$ is the Liouville-Vlasov operator. The source term $S$ accounts for production and disappearance of ions and electrons by ionization and recombination phenomena. According to $S$, a particle can be removed from an orbit, which is defined in terms of the constants of the motion as in the Vlasov theory $(S=0)$, also by radiation and collision phenomena. Electric and magnetic fields $\mathbf{E}=-\nabla \phi, \mathbf{B}=\nabla \times \mathbf{A}$ satisfy Maxwell equations with charge and current densities $e\left(\varrho_{+}-\varrho_{-}\right), \mathbf{j}=e\left(\mathbf{u}_{+} \varrho_{+}-\mathbf{u}_{-} \varrho_{-}\right)$given by $f_{ \pm}$. Exact solutions of this nonlinear system of integro-differential equations have been obtained for stationary conditions 
$(\partial / \partial t=0)$ on the $C S$ frame of reference (moving with a velocity $u_{0}$ with respect to the laboratory) by assuming constraints on $\varrho, \mathbf{u}, \mathbf{B}, \mathbf{E}$, and so on $S_{ \pm}, f_{ \pm}$, which are suggested by the experimental evidence (Nardi, 1970). Specifically the space variations along the filament axis are ignored (the filaments are considered essentially as parallel cylinders, $\partial / \partial z \approx 0)$ and in this direction $u_{z \pm}=-\varrho_{v \pm} c_{0}^{2} / \varrho_{ \pm} c_{\mp}$ where $c_{0}$ is the speed of light and $\varrho_{v \pm}$ is given by the orbit-controlled component $f_{v}$ of $f=f_{v}+f_{s}\left(\mathrm{~d} f_{v} / \mathrm{d} t=0\right.$; the $x$-axis is taken $\perp$ to $C S, z, y$ on $C S$ (see Figure 1 ) and so $\mathbf{B}_{\mathbf{y}} \approx \mathbf{B}_{\theta}$ ). Since by definition $\left|c_{ \pm}\right|=\left|\int f_{v \pm} v_{z} \mathrm{dv} / \varrho_{v \pm}\right|=$ constants $<c_{0}$, the contribution $\varrho_{s}$ to $\varrho$ from the source-controlled part $f_{s}$ must satisfy $\varrho_{ \pm}=\varrho_{v \pm}+\varrho_{s \pm}>\varrho_{v \pm} c_{0} /\left|c_{\mp}\right|$. This choice of $u_{z}$ is based on the criteria: (I) of coupling the mean (macroscopic) velocity of one species of particles with the other species with a maximum of simplicity and (II) to obtain self-consistent solutions of great generality, enough to match all constraints suggested by the experiments. Since $u_{z-}<0$ for a positive center electrode, $c_{+}>0 ; u_{z+}>0$ (for both polarities of the center electrode, see Bostick et al., 1970) so $c_{-}<0$. The self-consistent fields, flows, and densities are derived for $\varrho_{s+}=\varrho_{s-}, f_{v \pm}=\varrho_{0 \pm}\left(\alpha_{ \pm} m_{ \pm} / 2 \pi\right)^{3 / 2} \exp \left[-\alpha_{ \pm}\right.$ $\left.\left(\varepsilon_{ \pm}+c_{ \pm} p_{z \pm}+m_{ \pm} c_{ \pm}^{2} / 2\right)\right], \varepsilon_{ \pm}, p_{z \pm}=m_{ \pm} v_{z \pm} \pm e A_{z}$ are the energy and $z$-momentum of a particle. Our solutions are (emu):

$$
\begin{aligned}
A_{z} & =\left[e\left(c_{-}-c_{+}\right)\right]^{-1} \ln \left(F_{1}^{1 / \alpha_{+}} F^{1 / \alpha_{-}}\right) \\
\phi & =c_{+} \mid e\left(c_{+}-c_{-}\right) \ln \left(F_{1}^{c_{-} / c_{+}+\alpha_{+}} F_{2}^{1 / \alpha_{-}}\right) \\
& \text {where } F_{1}=\left[4 / \alpha_{+} K^{2}\right]\left|\partial g_{1} / \partial \eta\right|^{2}\left[1+\left|g_{1}\right|^{2}\right]^{-2},
\end{aligned}
$$

$g_{1}$ is an arbitrary (non-constant) function of the complex variable $\eta=x+i y$, $K^{2}=-e^{2} c_{0}^{2} \varrho_{0+}\left(c_{+}-c_{-}\right) 2 \pi / c_{-}$; by replacing in $F_{1}$ the constants $\alpha_{+}, \varrho_{0+}, c_{+}, c_{-}$ respectively with $\alpha_{-}, \varrho_{0-}, c_{-}, c_{+}$and $g_{1}$ with any non-constant $g_{2}$ we have $F_{2}$. The functions $= \pm e\left(\phi+c_{ \pm} A_{z}\right) \geqq 0$ have the role of effective potentials in $f_{v \pm}$ which represent jets of particles affecting the self-consistent field without directly entering short-range collision and ionization phenomena. The parameters $\alpha_{ \pm}, \varrho_{0 \pm}>0$ and $c_{ \pm}$play an essential role in the stability and decay of the filament system. Since the plasma is far from thermodynamical equilibrium, a temperature can be defined by $T_{ \pm}=\operatorname{trace} \int f_{ \pm}\left(\mathbf{v}-\mathbf{u}_{ \pm}\right)\left(\mathbf{v}-\mathbf{u}_{ \pm}\right) \mathrm{d} \mathbf{v} m_{ \pm} / 3 k \varrho_{ \pm}$rather than by $\left(k \alpha_{ \pm}\right)^{-1}(k=$ Boltzmann const.). Charge neutrality may or may not be imposed everywhere. If we take for simplicity $\varrho_{v+} / \varrho_{v-}=$ const., i.e. $F_{1} \sim F_{2}, \phi \sim A_{z}$ then the choice $g_{1}=g_{2}=a+\left(1+a^{2}\right)^{1 / 2}$ $e^{m \eta}$ gives $F \sim(\cosh m x+\operatorname{rcos} m y)^{-2}=\tau^{-2}, r=a\left(1+a^{2}\right)^{-1 / 2}$, which accounts for: (I) periodic arrangement of the filaments on the $y$-axis (simple period $=2 \pi / m$ ); (II) ionization high on $C S$, low far from $C S$, so $\varrho_{ \pm}$has maximum values on $C S$ at $x=0$, $\varrho_{ \pm}(x \rightarrow \pm \infty) \rightarrow 0$; (III) sharp increase of $B_{y}$ across $C S$, etc. Since $\partial / \partial z \approx 0, A_{z}$ determines completely $\boldsymbol{B}_{x}, \boldsymbol{B}_{y}$ i.e. $\mathbf{B}_{\boldsymbol{\tau}} \equiv \mathbf{B}-\mathbf{B}_{z}$. The velocity fields for electrons and ions in a filament region $(\tau<1+r)$ are given by $\mathbf{u}_{-}=\lambda(x, y) \mathbf{B}, \mathbf{u}_{\tau+}=\beta(x, y) \mathbf{B}_{\tau}$. The first condition accounts for the small electron inertia and $r_{e}$ (=electron Larmor radius) $<\lambda_{-}(=$electron mean free path) so that the electrons are tied to B (Lighthill, 1960). According to $\mathbf{u}_{+}(x \rightarrow-\infty) \rightarrow \mathbf{u}_{0}$ the ions have a kinetic energy which is mainly directed $\left(k T_{+} / \bar{\varepsilon}_{+k}<1 ; \bar{\varepsilon}_{+k}=\int\left(\varepsilon_{+}-e \phi\right) f_{+} \mathrm{d} \mathbf{v} / \varrho_{+}\right)$on $C S$ and for $x<0$. In spite of $r_{+}>\lambda_{+}$, we can 
consider then the mass-flow lines on $C S$ nearly coincident with the (non-adiabatic) orbit of an ion unaffected by collisions. The condition $\mathbf{u}_{\tau+}$ collinear with $\mathbf{B}_{\tau}$ is then a consequence of: (I) the observed invariance of the (equilibrium) filament configuration respect to sign $\left(\mathbf{u}_{\tau} \times \mathbf{B}_{\tau}\right)$ as it is shown by the apparently identical configurations of two filaments with opposite $\mathbf{B}_{z}$ and so opposite $\mathbf{u}_{\tau}$ : (II) the fact that any equilibrium with $\mathbf{u}_{\tau} \times \mathbf{B}_{\tau}=\mathbf{0}$ for which this invariance is automatically satisfied, is the simplest to describe analytically. The equations for $\lambda, \beta$ are given by the $x, y$ components of $\boldsymbol{\nabla} \times \mathbf{B}=\mathbf{j}$ (which are invariant for a simultaneous change of the signs of $\lambda, \beta$ ) with the solution $\lambda=\varrho_{v-} Q\left(A_{z}\right), \beta=\lambda+\left(c_{0}^{2} / e c_{+}\right) \mathrm{d}(1 / Q) / \mathrm{d} A_{z}$ where $Q$ is an arbitrary (nonconstant) function of $\boldsymbol{A}_{\mathbf{z}}$.

The field structure of two filaments with opposite $\mathbf{B}_{\mathbf{z}}$ components is represented in Figure 8. Each filament is a bundle of helical B-lines with a pitch increasing from the periphery to the axis of the filament $\left(B_{z}, \varrho_{ \pm}\right.$maximum on the axis). It is clear that other equilibrium configurations can be easily obtained by a different choice of $g_{1}, g_{2}$. If B, density, optical pattern, etc. from the experimental observation can be fitted by more than one solution, the choice of one self-consistent solution can be based on further constraints, e.g. the condition of greatest stability against modes growing exponentially with time. If we consider our solutions as the most stable against this

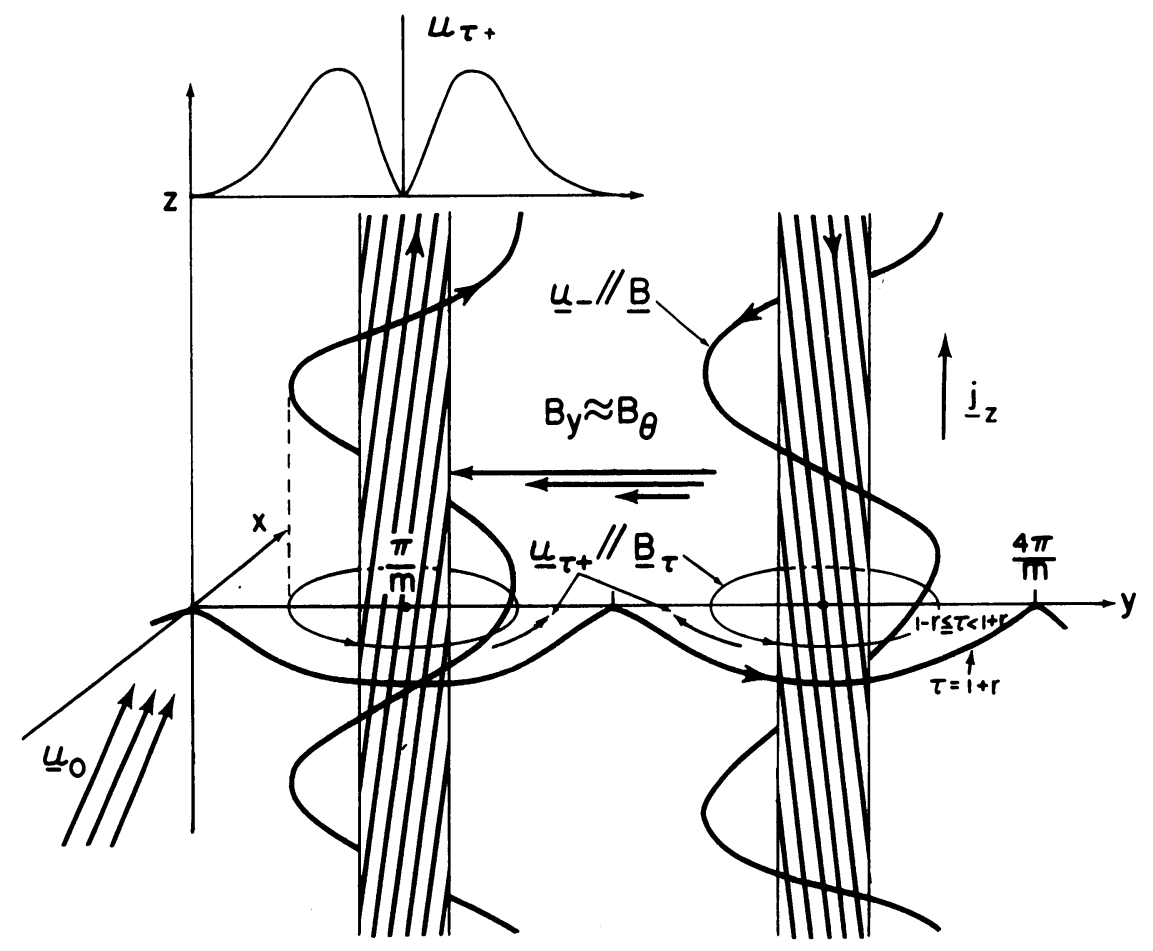

Fig. 8. Magnetic field lines and their projection $\left(A_{z}=\right.$ const.) on the $x, y$ plane in the filament region $\tau<1+r$. The magnitude $u_{\tau}$ of the ion mean velocity on the same region of the $x, y$ plane is plotted in the upper diagram (arbitrary scale). The mass flow $\left(\sim u_{0}\right)$ far ahead of $C S$ is parallel to the $x, z$ plane. 
kind of (secular) variations, we can still analyze the filament decay on a shorter time scale defined by some interval $\Delta t \ll T \approx 8 \mu$ s ( $=C A$ current period). Plasma and $C S$ are not an isolated system but the dynamic behavior on $\Delta t$ can be considered as determined mostly by the local plasma conditions. As an example, the collapse of $C S$ on $\Delta t_{1} \approx 0.1-$ $0.2 \mu$ s introduces time variations which cannot be considered of the same secular type as the $C A$ current period. This collapse as well as the related slowing-down of $C S$ in the off-axis region is responsible for flow and density rearrangements which trigger the decay (sometime explosive) of the filamentary structure. Explosive onsets of instabilities on a $\Delta t_{2} \ll \Delta t_{1}$ can be predicted by considering a whole series of continuous configurations of equilibrium (a Poincaré linear series, see Jeans, 1928) obtained by varying one of the parameters $\alpha_{ \pm}, \varrho_{0 \pm}, c_{ \pm}$.

These variations of the parameters on a time interval $\Delta t_{1}$ can be associated with the rearrangements due to collapse and slowing-down of CS. We have analized more in detail the linear series corresponding to different values of $c_{+}$. There is a critical value of $c_{+}$, depending also on the choice of $Q$, beyond which no stable configuration exists. This kind of instability could be related to the localized X-ray sources (see Figure 7) and to the appearance of bright spots on the filaments during the filament decay (corresponding to the flash phase of the flare). A detailed picture is supplied by our equilibrium configuration. Even a slight alteration in the collinearity of $\mathbf{u}_{-}$and $\mathbf{B}$ (eventually induced by an incoming jet of particles) can interrupt the $j_{z}$ current at a point of a filament. The consequence is the same as if the plasma there suddenly becomes essentially non conducting. The whole magnetic energy of the filament tends to be dissipated at that point so that the filament explodes (Alfvén and Carlquist, 1967).

A major part of the energy stored in the filamentary structure can be released by some other decay process (in correspondence with the main phase of the flare). The cyclotron resonant transfer of kinetic energy from filament-axis direction into the Larmor rotation (and vice-versa) can be considered as such a process ( $\mathrm{Nardi}, 1970$ ) along with the magnetic field annihilation between neighboring vortex filaments (Bostick et al., 1966; Gold and Hoyle, 1960) and other cooperative effects.

\section{Laboratory Experiment and Solar Flares}

The basic ingredients to produce the filamentary structure in the laboratory are: (I) a flow of dense highly-ionized gas $\left(10^{17}-10^{18}\right.$ particles $\left./ \mathrm{cm}^{3}\right)$ with a mean velocity $u_{0} \approx 10^{6}-10^{7} \mathrm{~cm} / \mathrm{s}$ from a region of low (or vanishing) magnetic field toward a region with a strong field $B_{\theta}\left(B_{\theta} \approx 10^{4}-10^{5} \mathrm{G}\right)$; (II) a steep gradient of this background field $B_{\theta}\left(\approx 10^{4}-10^{5} \mathrm{G} / \mathrm{cm}\right.$ between the two regions) in the direction of the velocity $\mathbf{u}_{0}$; (III) $\mathbf{u}_{0}$ nearly orthogonal to $\mathbf{B}_{\theta}$. In this case, a filamentary structure appears in the region of sharp transition; each filament axis is orthogonal to $\mathbf{B}_{\boldsymbol{\theta}}$ and in the plane containing $\mathbf{u}_{0}$. We recall that in the case, not considered here, of high field but small gradient (Bostick et al., 1966) or for relatively smaller values of $u_{0}$ (see Figure 4), filaments can be produced with the axis in the same direction of the background field $B_{\theta}$. The self-consistent field resulting from the interaction of the plasma with $B_{\theta}$ (in the 
transition region) has a fine structure which is underlined by the (visible light) filament arrangement. This self-consistent field region rests upon the high- $B_{\theta}$ region (without great variations with time) until an instability is triggered by some disturbance, e.g. a modulation in the incoming particle flow or a localized disruption. The decay of the fine-structure field with luminosity increase and particle acceleration can leave the background field $B_{\theta}$ substantially unchanged. Similar features can be found in solar flare phenomena. The field connecting solar regions (spots) of different polarity may correspond to our $\boldsymbol{B}_{\boldsymbol{\theta}}$. If an inward flux of particles flows over a region of predominantly horizontal field (i.e. near the null line of the observed longitudinal field) the formation of filaments can be expected. These fine-structure filaments (orthogonal to the background field) will appear in general as parallel to the isogauss lines (of the background longitudinal field). This seems to be in agreement with flare observations (Howard and Harvey, 1964). The inward motion can be observed by Balmer-line profile asymmetry (red wing brighter than blue wing in $90 \%$ of flares, Smith and Smith, 1963). Other observations by Severny (1958) indicate that field gradients near the neutral point are quite steep. The idea that only the filamentary fine-structure of the field decays during a flare is supported by the observation of flares for which no changes in the isogauss maps could be detected before and after the flare (Howard and Harvey, 1964). The flare $X$-ray spectrum in the range $20-100 \mathrm{keV}$ can be represented by a power law $N_{f}(\varepsilon) \sim \varepsilon^{-n}, 3 \leqq n \leqq 5$, (Fichtel and McDonald, 1967) which is essentially the same as the power law $N(\varepsilon) \sim \varepsilon^{-4 \pm 1}$ for the range $100-500 \mathrm{keV}$ from the plasma focus (Lee et al., 1970). These observations are perhaps sufficient to point out the useful role of laboratory experiments in the study of solar flares. The use of similarity transformations to gain a better insight in solar flare phenomena requires further work. It is a well-known fact that arc-plasma does not obey the same similarity laws available for other kinds of discharges.

\section{Acknowledgements}

Work supported by U.S. Air Force under Grant AFSOR-70-1842. (U.S.A.) and by Istituto Avogadro di Tecnologia, Roma C.P. 10757 (Italy).

\section{References}

Alfvén, H. and Carlquist, P.: 1967, Solar Phys. 1, 220.

Bernstein, M. J., Meskan, D. A., and van Paassen, H. L. L.: 1969, Phys. Fluids 12, 2193.

Bostick, W. H., Prior, W., Grunberger, L., and Emmert, G.: 1966, Phys. Fluids 9, 2078.

Bostick, W. H., Grunberger, L., Nardi, V., and Prior, W.: 1969, Proc. 9th Int. Conf. Ionized Gases, Bucharest, p. 66 .

Bostick, W. H., Grunberger, L. Nardi, V., and Prior, W.: 1970, Proc. 4th Int. Conf. Thermophys. Properties, Newton, Mass., p. 495.

Fichtel, C. E. and McDonald, F. B.: 1967, Ann. Rev. Astron. Astrophys. 5, 531.

Gold, T. and Hoyle, F.: 1960, Monthly Notices Roy. Astron. Soc. 120, 89.

Howard, R. and Harvey, J. W.: 1964, Astrophys. J. 139, 1328.

Jeans, J.: 1928, Astronomy and Cosmogony, Cambridge University Press., Cambridge, England, Ch. 7. 
Lee, J. H., Conrads, H., Williams, M. D., Shomo, L. P., and Hermansdorfer, H.: 1968, Bull. A.P.S. 13, 1543.

Lee, J. H., Loebbaka, D. S., and Roos, C. E.: 1970, Bull. A.P.S. 15, 642.

Lighthill, M. J.: 1960, Phil. Trans. A252, 397.

Marshall, J.: 1960, Phys. Fluids 3, 134.

Mather, J. W.: 1965, Phys. Fluids 8, 366.

Nardi, V.: 1970, Phys. Rev. Letters 25, 718.

Patou, C., Simmonet, A., and Watteau, J. P.: 1969, Phys. Letters 29A, 1.

Severny, A. B.: 1958, Izv. Krymsk. Astrofiz. Obs. $20,22$.

Smith, H. J. and Smith, E. P.: 1963, Solar Flares, Ch. 6.

\section{Discussion}

Brueckner: What is the density ratio in the center of the arc prior to and during the collapse?

Nardi: Our initial $D_{2}$ density was $2.8 \times 10^{17} \mathrm{~cm}^{-3} ; 10^{19} \mathrm{~cm}^{-3}$ can be accepted as the correct order of magnitude of the maximum density of electrons ( $\cong$ ion density) after current sheath collapse during the neutron pulse. Different methods (Schlieren, best fit to the bremsstrahlung, etc.) point to this value. A maximum value $>4 \times 10^{18} \mathrm{~cm}^{-3}$ for electrons has been obtained just before the beginning of the neutron pulse, by measurement of $90^{\circ}$ cooperative scattering of a ruby-laser beam by the plasma (coaxial accelerator and operating conditions were comparable to ours; J. P. Baconnet et al., Proc. Ninth Int. Conf. Ionized Gases, Bucharest 1969, p. 643). Our measurements (by H $\beta$ Stark broadening) of the electron density in the filaments, before collapse of the current sheath, gives $1-2 \times 10^{18} \mathrm{~cm}^{-3}$, i.e. $\sim$ three times the initial density.

Wilcox: Referring to the slide (Figure 2) why do the radial filaments pair up with the current and field in opposite directions in each member of a pair?

Nardi: The ions can move across the magnetic field with a Larmor radius of the same order as the filament optical radius, whereas the electrons are tied to the field. Therefore a distinction must be made about the different components of current density $\mathbf{j}$. The current carriers are different in different directions. The $z$-component $j_{z}$, along the filament axis, is mainly carried by the electrons (this is indicated by specific experimental data, see W. H. Bostick et al., Proc. Fifth Symp. Thermophysical Properties, Newton, Mass. 1970, Amer. Soc. Mech. Eng. edit., p. 495) and has the same direction in all filaments with a positive or a negative value depending only on the electrode polarity. The corresponding $\left(\boldsymbol{B}_{y}, \boldsymbol{B}_{x}\right)$ field lines in a filament region are closed loops with center on the filament axis, having a common direction defined by sign $\left(j_{z}\right)$. On the other hand, in the $(x, y)$ plane, orthogonal to the current sheath driven by the electrode applied field, the ions are the current carriers. This becomes clear by considering: (I) the electron inertia as negligible if compared with ion inertia and (II) net charge density as very small compared with the electron density. Then it can be easily shown (M. J. Lighthill, Phil. Trans. Roy. Soc. A252, 397 (1960)) that a low rate of loss of electron momentum, per unit volume, by collision with ions implies that the magnetic lines of force 'move with' or are 'frozen into' the electron gas rather than 'with' or 'into' the flowing mass. This is our case and it can be considered an accurate picture whenever the magnetic field is so high that the Hall-current effect is much more important than the electrical resistivity (i.e. the gyro-frequency of electrons greatly exceeds their collision frequency).

Since the electrons are tied to the magnetic field lines, in the current sheath frame of reference the ions carry the current in any direction orthogonal to these lines (i.e., essentially in the $(x, y)$ plane since $B_{z}$ is by far the largest component in a filament region). For our self-consistent solutions the projection on the plane $(x, y)$ of the mass flow lines corresponding to the vortex structure of each filament, are closed loops nearly, or exactly, collinear (depending on our choice) with the $\left(B_{x}, B_{y}\right)$ field loops. Solutions exist corresponding to both parallel or antiparallel motions with respect to these field loops. The mass flow which minimizes the shear, however, corresponds to a vorticity with opposite directions in neighboring filaments and is, likely, the preferred one by the plasma. Opposite rotational velocity of the ions implies opposite currents in the $(x, y)$ plane and so opposite components $B_{z}$ of the self-consistent field. The paired arrangement of the filaments can be considered then as corresponding to the most stable configuration with a minimum shear in the velocity field. 